\title{
RESEARCH
}

Open Access

\section{Selective deletion of hepatocyte platelet- derived growth factor receptor $a$ and development of liver fibrosis in mice}

Beom Jin Lim ${ }^{1+}$, Woon-Kyu Lee ${ }^{2+} \mathbb{D}$, Hyun Woong Lee ${ }^{3}$, Kwan Sik Lee ${ }^{3}$, Ja Kyung Kim³ ${ }^{3}$ Hye Young Chang ${ }^{4}$ and Jung II Lee Le $^{3^{*}}$

\begin{abstract}
Background: Platelet-derived growth factor receptor a (PDGFRa) expression is increased in activated hepatic stellate cells (HSCs) in cirrhotic liver, while normal hepatocytes express PDGFRa at a negligible level. However, cancerous hepatocytes may show upregulation of PDGFRa, and hepatocellular carcinoma is preceded by chronic liver injury. The role of PDGFRa in non-cancerous hepatocytes and liver fibrosis is unclear. We hypothesized that upon liver injury, PDGFRa in insulted hepatocytes contributes to liver fibrosis by facilitating intercellular crosstalk between hepatocytes and HSCS.
\end{abstract}

Methods: Hepatocytes were isolated from normal and thioacetamide (TAA)-induced cirrhotic livers for assessment of PDGFRa expression. Conditional knock-out (KO) C57BL/6 mice, in which PDGFRa was selectively deleted in hepatocytes, were generated. Liver fibrosis was induced by injecting TAA for 8 weeks. Hep3B cells were transfected with a small interfering RNA (siRNA) (PDGFRa or control) and co-cultured with LX2 cells.

Results: PDGFRa expression was increased in hepatocytes from fibrotic livers compared to normal livers. Conditional PDGFRa KO mice had attenuated TAA-induced liver fibrosis with decreased HSC activation and proliferation. Immunoblot analyses revealed decreased expression of phospho-p44/42 MAPK in TAA-treated KO mice; these mice also showed almost complete suppression of the upregulation of mouse double minute 2. Although KO mice exhibited increased expression of transforming growth factor (TGF)- $\beta$ and Smad2/3, this was compensated for by increased expression of inhibitory Smad7. LX2 cells co-cultured with PDGFRa siRNA-infected Hep3B cells showed decreased PDGFRa, a smooth muscle actin, collagen a1(I), TGF $\beta$, and Smad2/3 expression. LX2/ PDGFRa-deleted hepatocyte co-culture medium showed decreased PDGF-BB and PDGF-CC levels.

Conclusions: Deletion of PDGFRa in hepatocytes attenuated the upregulation of PDGFRa in HSCs after TAA treatment, resulting in decreased liver fibrosis and HSC activation. This suggests that in the event of chronic liver injury, PDGFRa in hepatocytes plays an important role in liver fibrosis by affecting PDGFRa expression in HSCs.

Keywords: Platelet-derived growth factor receptor a, Liver fibrosis, Liver cirrhosis, Hepatocyte, Hepatic stellate cell

\footnotetext{
* Correspondence: mdflorence@yuhs.ac

${ }^{\dagger}$ Beom Jin Lim and Woon-Kyu Lee contributed equally to this work.

${ }^{3}$ Department of Internal Medicine, Gangnam Severance Hospital, Yonsei

University College of Medicine, Seoul, Republic of Korea

Full list of author information is available at the end of the article
}

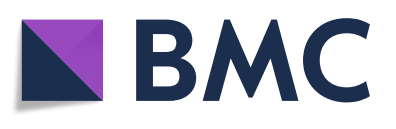

(c) The Author(s). 2018 Open Access This article is distributed under the terms of the Creative Commons Attribution 4.0 International License (http://creativecommons.org/licenses/by/4.0/), which permits unrestricted use, distribution, and reproduction in any medium, provided you give appropriate credit to the original author(s) and the source, provide a link to the Creative Commons license, and indicate if changes were made. The Creative Commons Public Domain Dedication waiver (http://creativecommons.org/publicdomain/zero/1.0/) applies to the data made available in this article, unless otherwise stated. 


\section{Background}

Liver fibrosis is a multicellular response to liver injury in which hepatic stellate cells (HSCs) are responsible for $90 \%$ of extracellular matrix (ECM) production [1-5]. Liver fibrosis upon acute liver injury may be beneficial by providing mechanical stability and facilitating the restoration of hepatic architecture and function. However, repetitive chronic liver insults result in liver fibrosis, which may progress to cirrhosis $[3,6,7]$, the major contributing factor to development of hepatocellular carcinoma (HCC) [8].

Upon liver injury, HSCs are activated by cytokines, including platelet-derived growth factors (PDGFs), which are potent mitogens $[9,10]$. PDGFs exist as five isoforms: PDGF-AA, PDGF-AB, PDGF-BB, PDGF-CC, and PDGF-DD. The cellular effects of PDGFs require the function of cell surface receptor tyrosine kinases comprising platelet-derived growth factor receptor (PDGFR) $\alpha$ and PDGFR $\beta$ subunits $[11,12]$. Elevated PDGFR expression is observed in human cardiac fibrosis after myocardial infarct, pulmonary fibrosis, kidney fibrosis, and liver fibrosis [13-16].

PDGFR expression is very low in the normal adult liver but is significantly upregulated in HSCs in cirrhotic liver [17]. PDGFR $\alpha$ participates in liver fibrosis, as evidenced by increased PDGFR $\alpha$ expression in cirrhotic human livers, human cell lines, and a mouse model of liver fibrosis $[18,19]$. Stimulation of PDGFR $\alpha$ expression in HSCs induces their activation and proliferation [16, 20-23], and blocking of PDGFR $\alpha$ expression suppresses HSC proliferation [24]. In addition, stimulation of PDGFR $\alpha$ expression by excessive production of PDGF-CC induces liver cirrhosis and HCC in PDGF-C transgenic (Tg) mice [25, 26]. PDGFR $\alpha$ expression in the liver is markedly increased in PDGF-C Tg mice, although the responsible cell type is unclear.

Normal adult hepatocytes show negligible PDGFR $\alpha$ expression. However, hepatocytes in embryos may have enhanced PDGFR $\alpha$ expression, which later is markedly attenuated [27]. Therefore, the role of PDGFR $\alpha$ expression in adult hepatocytes in liver fibrosis is unknown. However, the increased PDGFR $\alpha$ expression in HCC subsets in the absence of background liver cirrhosis suggests that abnormal hepatocytes overexpress PDGFR $\alpha$ [28].

Our preliminary study demonstrated that PDGFR $\alpha$ expression was increased in not only stimulated HSCs but also injured hepatocytes. We hypothesized that PDGFR $\alpha$ in hepatocytes in the setting of chronic liver injury plays an important role in liver fibrosis by facilitating intercellular crosstalk between hepatocytes and HSCs.

We report for the first time that conditional abolition of PDGFR $\alpha$ expression in hepatocytes attenuates chemically induced liver fibrosis by suppressing the upregulation of PDGFR $\alpha$ and TGF $\beta$ in HSCs, reducing their activation and proliferation.

\section{Methods}

Generation of PDGFRa conditional KO mice

Homozygous PDGFR $\alpha^{\text {loxp/Loxp }}$ mice with targeted deletion of exons 1 to 4 and albumin (alb)-Cre mice, both on the C57BL/6 background, were obtained from Jackson Laboratories (Bar Harbor, ME). PDGFR $\alpha^{\text {loxp/Loxp }}$ mice were crossbred with Alb-Cre mice to obtain offspring carrying PDGFR $\alpha^{\text {loxp/wt }}$;Alb-Cre. Next, PDGFR $\alpha^{\text {loxp/wt }}$;AlbCre were bred to PDGFR $\alpha^{\operatorname{loxp} / \text { Loxp }}$ mice, resulting in PDGFR $\alpha^{\text {loxp/Loxp }} ; \mathrm{Alb}-\mathrm{Cre}^{+/-}$or $\mathrm{KO}$ mice. Mice of all other genotypes, including PDGFR $\alpha^{\text {loxp/Loxp }}$ Alb- $\mathrm{Cre}^{-/-}$and

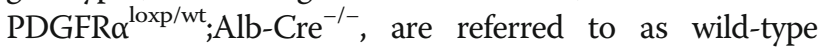
(WT) controls.

\section{Animals and thioactetamide-induced liver fibrosis}

All experiments on mice were approved by the Institutional Animal Use and Care Committee (IACUC) at Gangnam Severance Hospital, Yonsei University College of Medicine (permit number 0031) in accordance with the recommendations and restrictions of the IACUC, which follows the guidelines of the United States National Institutes of Health.

Male 8-week old C57BL/6 (WT) and PDGFR $\alpha$ conditional KO mice (PDGFR $\alpha \mathrm{KO}$ ) were used. The animals were housed with a $12 \mathrm{~h}$ light:dark cycle and fed standard chow. For the fibrosis groups, thioacetamide (TAA) (300 $\mathrm{mg} / \mathrm{kg}$ body weight) was injected intraperitoneally into male WT and PDGFR $\alpha$ KO mice during the light cycle twice weekly for 8 weeks. One week after the final TAA treatment, the mice were fasted for $14-16 \mathrm{~h}$ with free access to water and subsequently euthanized. Untreated age-matched WT or PDGFR $\alpha$ KO male mice were euthanized after the fast.

The animal experiment groups were as follows: WTcontrol $(n=5)$, untreated WT mice; KO-control $(n=5)$, untreated PDGFR $\alpha$ conditional KO mice; WT-TAA $(n=5)$, WT mice treated with TAA for 8 weeks; and KO-TAA $(n=5), P D G F R \alpha$ conditional $\mathrm{KO}$ mice treated with TAA for 8 weeks.

\section{Hepatocyte and HSC isolation}

Hepatocytes and HSCs were isolated from WT and WT-TAA mice by an in situ collagenase perfusion method as described previously with modifications [29]. Protein and RNA were extracted from the isolated hepatocytes for analyses.

\section{HCC tissue}

Freshly frozen liver-tissue specimens from livers resected due to $\mathrm{HCC}$ or metastatic liver cancer were used. The liver specimens were archived at the Tissue Bank of Yonsei University College of Medicine, Gangnam Severance Hospital with the patients' consent. The non-tumor sites of liver specimens with hepatitis but not 
liver cirrhosis as determined via pathologic evaluation $(n=7)$ were compared to liver specimens from metastatic liver cancer patients $(n=7)$ that appeared normal on pathologic assessment. This study was approved by the Institutional Review Board of Gangnam Severance Hospital, Yonsei University College of Medicine, Seoul, Republic of Korea (3-2015-0045).

\section{Immunohistochemical analyses and terminal dUTP nick end-labeling assay}

Liver-tissue specimens were stained with Masson's trichrome and the fibrosis ratio was calculated using an image analysis system as described previously with modifications [30]. The total area was calculated as the sum of the area of the microscopic fields, including parenchyma and fibrosis. For each slide, the area of fibrosis was evaluated in 20 consecutive fields at a magnification of $\times 200$, and averaged.

Sections of mouse liver tissue specimens were immunostained using a mouse anti-human alpha-smooth muscle actin ( $\alpha$ SMA) primary antibody (DAKO, Carpinteria, CA). Detection of the primary antibody was carried out by the immunoperoxidase technique using an ABC Kit (Vector Laboratories) as a measure of HSC activation. Peroxidase activity was determined by reaction with diaminobenzidine tetrahydrochloride (DAB). Data are total numbers of $\alpha$ SMA-positive cells present in 10 high-power fields $(\times 400)$ per sample.

Apoptosis was evaluated by terminal dUTP nick end-labeling (TUNEL) assay. The TUNEL assay detects fragmentation of nuclear DNA during apoptotic cell death in situ, and was performed using an ApopTag Peroxidate In Situ Apoptosis Detection Kit (Millipore, USA). For each slide, the area of immunostained apoptotic nuclei by 3'-OH-end labeling of fragmented DNA was evaluated in 20 consecutive fields at a magnification of $\times 200$, and averaged.

Sections of human liver-tissue specimens were immunostained with a mouse anti-PDGFR $\alpha$ antibody (Santa Cruz Biotechnology, Santa Cruz, CA) and assessed at a magnification of $\times 400$.

\section{siRNA transfection}

Transient knockdown assays were performed using DharrmaFECT 1 (Dharmacon, Lafayette, CO), according to the manufacturer's instructions. PDGFR $\alpha$ (\#5156) and control (D-001206-13) siRNAs were purchased from Dharmacon.

\section{Cell lines and in vitro experiments}

Hep3B (KCLB \#88064) and LX2 (cat \#SCC064) cells were purchased from the Korean Cell Line Bank (Seoul, Republic of Korea), and EMD Millipore (Temecula, $\mathrm{CA})$, respectively, and maintained as described previously [31, 32].
Cells were cultured using hanging cell-culture inserts ( $1 \mu \mathrm{m}$ pore size, Falcon) to separate cell populations. Wells and inserts with medium were allowed to stabilize for $24 \mathrm{~h}$ at $37^{\circ} \mathrm{C}$ according to the manufacturer's recommendations. Hep3B cells were seeded in the insert $\left(3 \times 10^{3}\right.$ cells/ $\mathrm{cm}^{2}$ ) and allowed to attach overnight in Dulbecco's modified Eagle's medium (DMEM) with 10\% fetal bovine serum (FBS). The following day, siRNA (PDGFR $\alpha$ or control) was transfected into Hep3B cells using DharmaFECT 1 (Dharmacon, Lafayette, CO), according to the manufacturer's instructions. Briefly, LX2 cells $\left(3 \times 10^{5} / \mathrm{cm}^{2}\right)$ were seeded on a six-well plate and incubated for $24 \mathrm{~h}$. Hep3B cells were plated separately on a culture insert and transfected with the PDGFR $\alpha$ or control siRNA. After a $6 \mathrm{~h}$ stabilizing period, the insert containing treated Hep3B cells was placed above the plate with LX cells and incubated for a further $24 \mathrm{~h}$. LX2 Cells and supernatants were harvested and recovered for further analyses.

\section{Cell viability assay}

The 3-(4,5-dimethylthiazol-2-yl)-2,5-diphenyltetrazolium bromide (MTT) assay was used to evaluate the proliferation of LX cells. Following co-culture for $24 \mathrm{~h}, 5 \mathrm{mg} / \mathrm{mL}$ MTT was added to the culture medium (Sigma-Aldrich, St. Louis, MO) and incubated for $4 \mathrm{~h}$ at $37^{\circ} \mathrm{C}$. The supernatant was removed, the cells were treated with $150 \mu \mathrm{L} /$ well dimethyl sulfoxide, and the absorption at $470 \mathrm{~nm}$ was measured. The assay was carried out in triplicate and each experiment was repeated at least three times. Data are percentages of surviving cells relative to the control.

\section{RNA isolation and real-time polymerase chain reaction}

Total RNA was extracted from frozen whole livers or isolated cells using TRIzol reagent (Invitrogen, Carlsbad, CA) or Qiagen mini columns (Qiagen Inc. Valencia, CA) according to the manufacturer's protocol. RNA concentrations were quantified by spectrophotometry. RNA integrity was assessed by agarose gel electrophoresis and ethidium bromide staining. The RNA samples were diluted in RNase-free water and stored at $-70^{\circ} \mathrm{C}$ until use. Five micrograms of RNA were reverse-transcribed using the RNA PCR Kit, version 1.2 (TaKaRa Bio Inc., Japan) according to the manufacturer's recommendations. Oligonucleotide primers and a TaqMan probe for PDGFR $\alpha$;GF- $\beta$; Smad 2,3 , and 7 ; collagen $\alpha 1(I)$ $(\operatorname{Col} 1 \alpha(I)) ; \alpha S M A ; b a x ; b c l-2 ;$ and MDM2 were used, with $18 S$ as the internal control. The probes were obtained from Applied Biosystems (Perkin-Elmer/PE Applied Biosystems, Foster City, CA). The TaqMan probe was labeled at the $5^{\prime}$-end with the reporter dye FAM and at the 3 '-end with a minor groove binder (MGB) nonfluorescent quencher. Quantitative polymerase chain reaction (qPCR) was performed in triplicate 
for each sample on a Step One Plus Real Time System (Applied Biosystems). Each $20 \mu \mathrm{L}$ reaction contained $10 \mu \mathrm{L}$ TaqMan Fast Universal Master Mix (Applied Biosystems, Darmstadt, Germany), $1 \mu \mathrm{L}$ Gene Expression Mix, and $2 \mu \mathrm{L} c \mathrm{cNA}$ diluted in $7 \mu \mathrm{L}$ RNase-free water. The thermal cycler conditions were $20 \mathrm{~s}$ at $95^{\circ} \mathrm{C}$, followed by 40 cycles of $5 \mathrm{~s}$ at $95^{\circ} \mathrm{C}$ and $20 \mathrm{~s}$ at $60^{\circ} \mathrm{C}$. Fold changes in the expression of target genes relative to the endogenous $18 \mathrm{~S}$ control were calculated as described previously [33].

\section{Protein extraction and immunoblotting}

Whole-liver lysates were prepared in Triton-X 100 lysis buffer containing protease inhibitors and protein concentrations were quantified using the Bradford method with bovine serum albumin (BSA) as the standard. Resolution by SDS-PAGE was followed by immunoblotting using the following antibodies: rabbit anti-PDGFR $\alpha$ (\#3164), rabbit anti-phospho-p44/42 MAPK (\#9101), rabbit anti-p44/42 MAPK (\#9102), rabbit anti- $\beta$ actin (\#4(a967), all from Cell Signaling (Danvers, MA) and anti-phospho-Smad3 (\#ab52903) from Abcam (Cambridge, MA). Epitope-primary antibody complexes were detected using species-specific secondary antibodies conjugated to horseradish peroxidase (HRP) followed by enhanced chemiluminescence (ECL) (Thermo Fisher Scientific Pierce, IL).

\section{Determination of PDGF-CC and -BB levels in culture} medium

The levels of PDGF-CC and PDGF-BB in culture medium were quantified using a Quantikine enzyme-linked immunosorbent assay (ELISA) Kit (R\&D Systems, Minneapolis, MN) according to the manufacturer's instructions.

\section{Statistical analyses}

Results are shown as means \pm standard errors of the mean (SEMs). Data were subjected to nonparametric analyses (Kruskal-Wallis or Mann-Whitney test) or one-way analysis of variance (ANOVA) with Tukey's post hoc test. A value of $P<0.05$ was considered indicative of statistical significance. All calculations were performed in IBM SPSS Statistics version 23 for Windows.

\section{Results}

PDGFRa deletion in hepatocytes attenuated the TAAinduced increase in PDGFRa expression in the whole liver The level of PDGFR $\alpha$ expression is increased in fibrotic liver due to its overexpression in HSCs [19, 34]. Noninsulted hepatocytes scarcely express PDGFR $\alpha$ [19].

Hepatocytes from TAA-treated livers showed increased PDGFR $\alpha$ expression compared to those from normal livers (Fig. 1a). To address the role of hepatocyte PDGFR $\alpha$ in liver fibrosis, we generated hepatocyte-specific
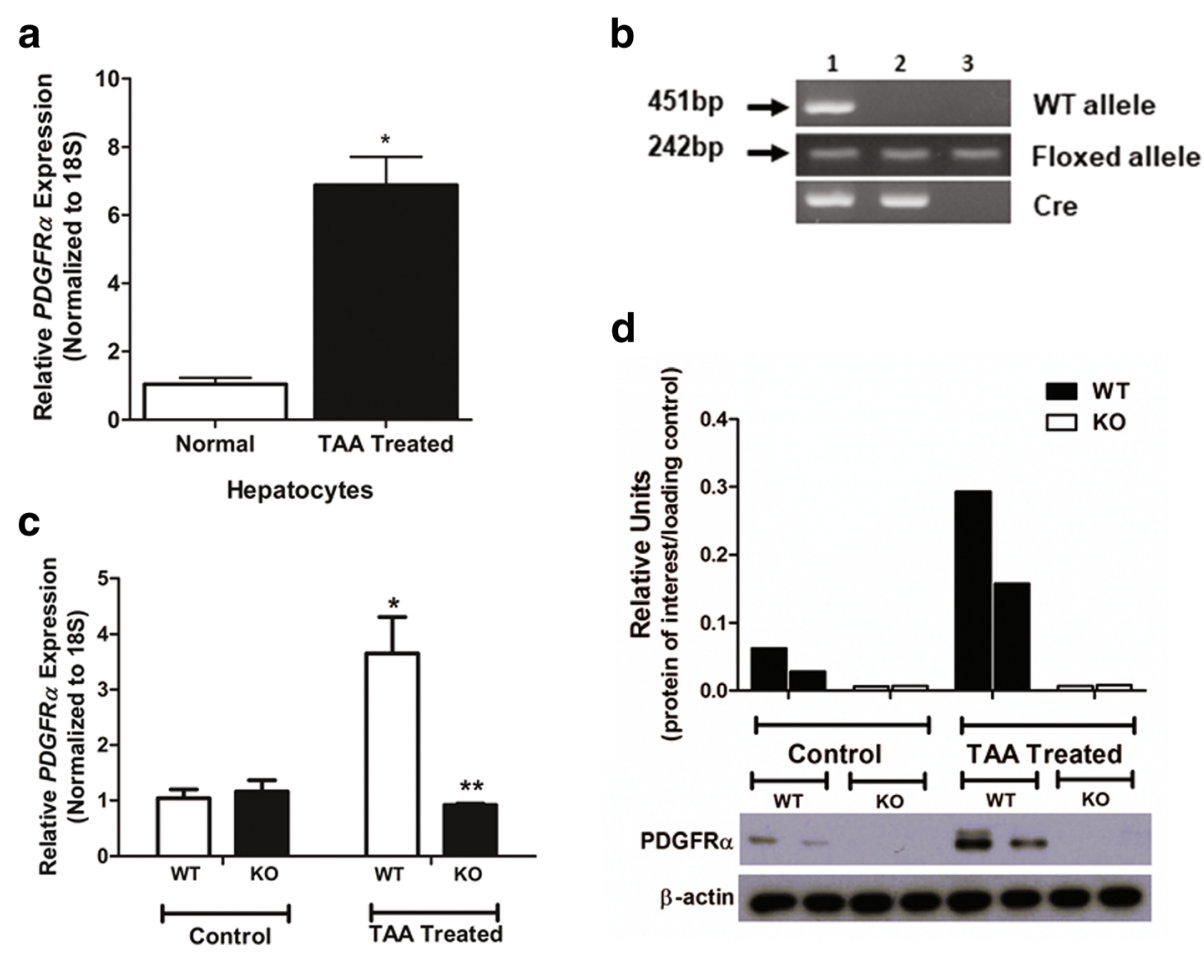

Fig. 1 Attenuation of PDGFRa expression in TAA-treated livers after conditional deletion of PDGFRa in hepatocytes. a PDGFRa in hepatocytes. b Identification of hepatocyte PDGFRa-deleted knockout (KO) mice by genotyping PCR (lane 2). c PDGFRa expression in the liver of wild-type (WT) and hepatocyte PDGFRa-deleted KO mice (d) Western blotting analyses; densitometry values normalized to that of $\beta$-actin are shown. ${ }^{*} P<0.05$, compared to that of TAA-untreated WT mice. ${ }^{*} P<0.05$, compared to WT mice under identical conditions 
conditional PDGFR $\alpha$ KO mice (Fig. 1b). The non-insulted livers (control) of WT and conditional $\mathrm{KO}$ mice showed no significant differences in PDGFR $\alpha$ expression (Fig. 1c). However, the livers of TAA-treated WT mice, but not those of TAA-treated KO mice, had enhanced PDGFR $\alpha$ expression (Fig. 1c).

Immunoblot analyses of the whole liver showed an increased PDGFR $\alpha$ protein level in TAA-treated WT mice, and a significantly reduced PDGFR $\alpha$ protein level in TAA-treated $\mathrm{KO}$ mice (Fig. 1d).

\section{PDGFRa deletion in hepatocytes alleviated TAA-induced} HSC activation and liver fibrosis

The fibrotic area in the liver was increased by TAA in both WT and KO mice (Fig. 2a). However, PDGFR $\alpha$ conditional $\mathrm{KO}$ mice showed significantly attenuated liver fibrosis compared to WT mice (Fig. 2a). Quantitative analyses of $\operatorname{col} 1 \alpha(I)$ expression yielded a result comparable with the histologic findings (Fig. 2b).

The TAA-induced increase in the expression of $\alpha S M A$, a marker of HSC activation, was significantly abolished by deletion of PDGFR $\alpha$ (Fig. 2c). The number of $\alpha S M A-p o s i t i v e$ cells was significantly increased and decreased by TAA in the liver of WT and KO mice, respectively (Fig. 2d).

\section{Deletion of PDGFRa in hepatocytes attenuates ERK activation}

ERK is a downstream signaling factor involved in PDGFinduced activation of PDGFR [35]. Immunoblot analyses of phospho-p44/42 MAPK (ERK1/2) revealed increased phosphorylation of ERK1/2 in the livers of WT and KO TAA-treated mice compared to the controls. However,

\section{a}
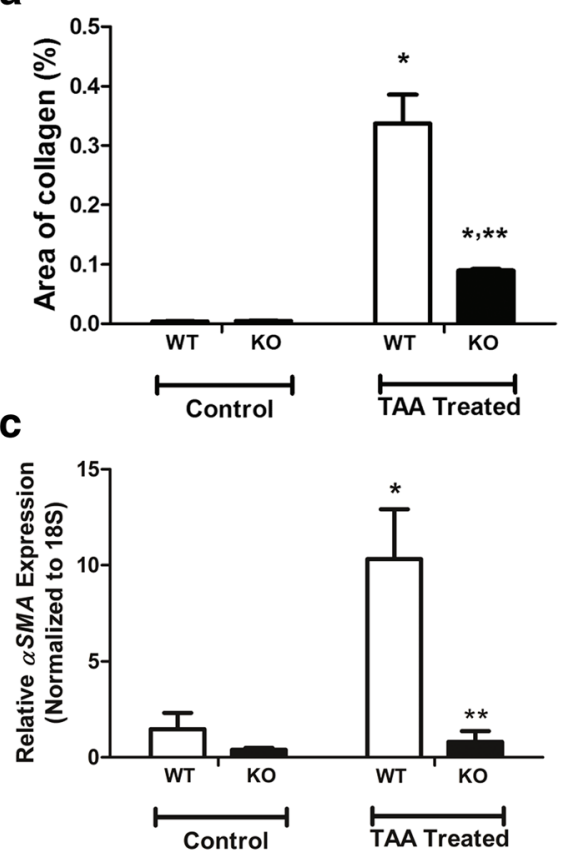

d

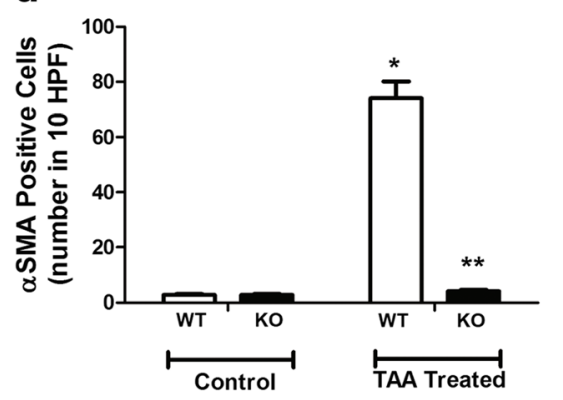

b

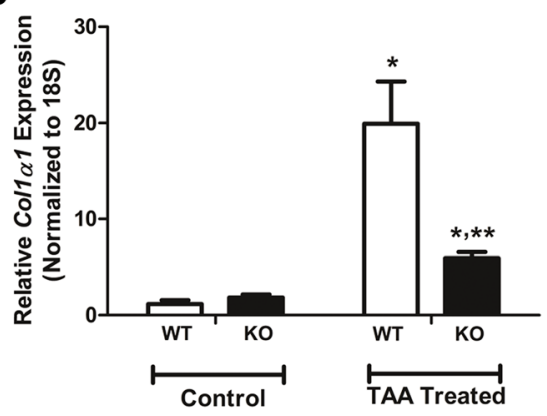

e

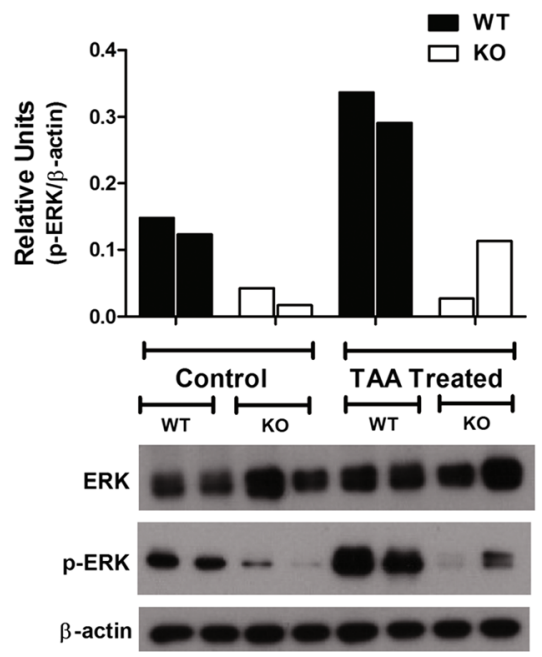

Fig. 2 Suppression of TAA-induced liver fibrosis by hepatocyte platelet-derived growth factor receptor (PDGFR) a deletion. a Area of fibrosis. b Expression of (b) Colla(l) and c aSMA. d Number of aSMA-positive cells. e Western blotting analyses; densitometry values are shown. ${ }^{*} P<0.05$, compared to TAA-untreated WT mice. ${ }^{* *} P<0.05$, compared to WT mice under identical conditions 
$\mathrm{KO}$ mice showed significantly attenuated phosphorylation of ERK1/2 compared to WT TAA-treated mice (Fig. 2e).

\section{Deletion of PDGFRa did not influence hepatocyte apoptosis}

TAA treatment increased the number of cells displaying nuclear fragmentation compared to the control. Deletion of PDGFR in hepatocytes did not affect apoptosis irrespective of TAA treatment, similar to the WT control (Fig. 3a, b). Bcl-2 inhibits apoptosis whereas Bax promotes it [36], and the $\mathrm{Bcl}-2 / \mathrm{Bax}$ ratio is a marker of survival after apoptotic stimuli [37]. The liver $\mathrm{Bcl}-2 / \mathrm{Bax}$ ratio did not differ significantly between WT and KO mice after TAA treatment (Fig. 3c).

\section{Effect of hepatocyte PDGFRa deletion on TGF $\beta$ signaling} Activation of PDGFR by excessive PDGF ligands results in upregulation of the TGF $\beta /$ Smad signaling pathway to facilitate HSC proliferation and collagen production [38]. In this study, deletion of PDGFR $\alpha$ in hepatocytes resulted in increased TGF $\beta$ expression in normal and TAA-treated livers compared to the WT (Fig. 4a). Downstream signaling of TGF $\beta$ and $S m a d 2 / 3$ was also upregulated by deletion of PDGFR $\alpha$ in hepatocytes in both normal and TAA-induced fibrotic livers compared to the WT (Fig. 4b, c). The protein level of Smad3 showed a similar trend to the mRNA level (Fig. 4d). Smad7 was significantly upregulated in the liver of conditional PDGFR $\alpha$-deleted mice; this likely compensated for the effects of enhanced TGF- $\beta$ signaling (Fig. 4d).
Deletion of PDGFRa in hepatocytes blocked upregulation of MDM2

MDM2 is a negative regulator of p53 and is considered an oncogene $[39,40]$. Activation of PDGR $\alpha$ independently of PDGF results in activation of MDM2 and suppression of p53 [41, 42]. TAA-induced liver injury was accompanied by upregulation of $M D M 2$ in the liver; this was blocked by deletion of PDGFR $\alpha$ in hepatocytes (Fig. 5a).

To speculate on the cellular components responsible for the TAA-induced increase in $M D M 2$ expression, hepatocytes and HSCs were isolated from TAA-treated livers and MDM2 expression was evaluated. Although both hepatocytes and HSCs from injured livers showed increased MDM2 expression, the magnitude of the upregulation was significantly greater in hepatocytes than in HSCs (Fig. 5b).

\section{Co-culture with PDGFRa-deleted Hep3B cells decreased PDGFRa expression in, and suppressed the activation of, LX2 cells}

Hep3B cells have elevated PDGFR $\alpha$ expression [19, 43]. Culture of LX2 cells with PDGFR $\alpha$-silenced Hep3B cells attenuated PDGFR $\alpha$ expression in the former (Fig. 6a). They had decreased $\alpha S M A$ and coll $\alpha(I)$ expression compared to that on LX2 cells, co-cultured with Hep3B cells with normally expression PDGFR $\alpha$ (Fig. 6b, c).

LX2 cells were co-cultured with PDGFR $\alpha$-silenced or control Hep3B cells for $24 \mathrm{~h}$. The proliferation of LX2 cells was reduced by co-culture with $P D G F R \alpha$-silenced Hep3B cells (Fig. 6d).

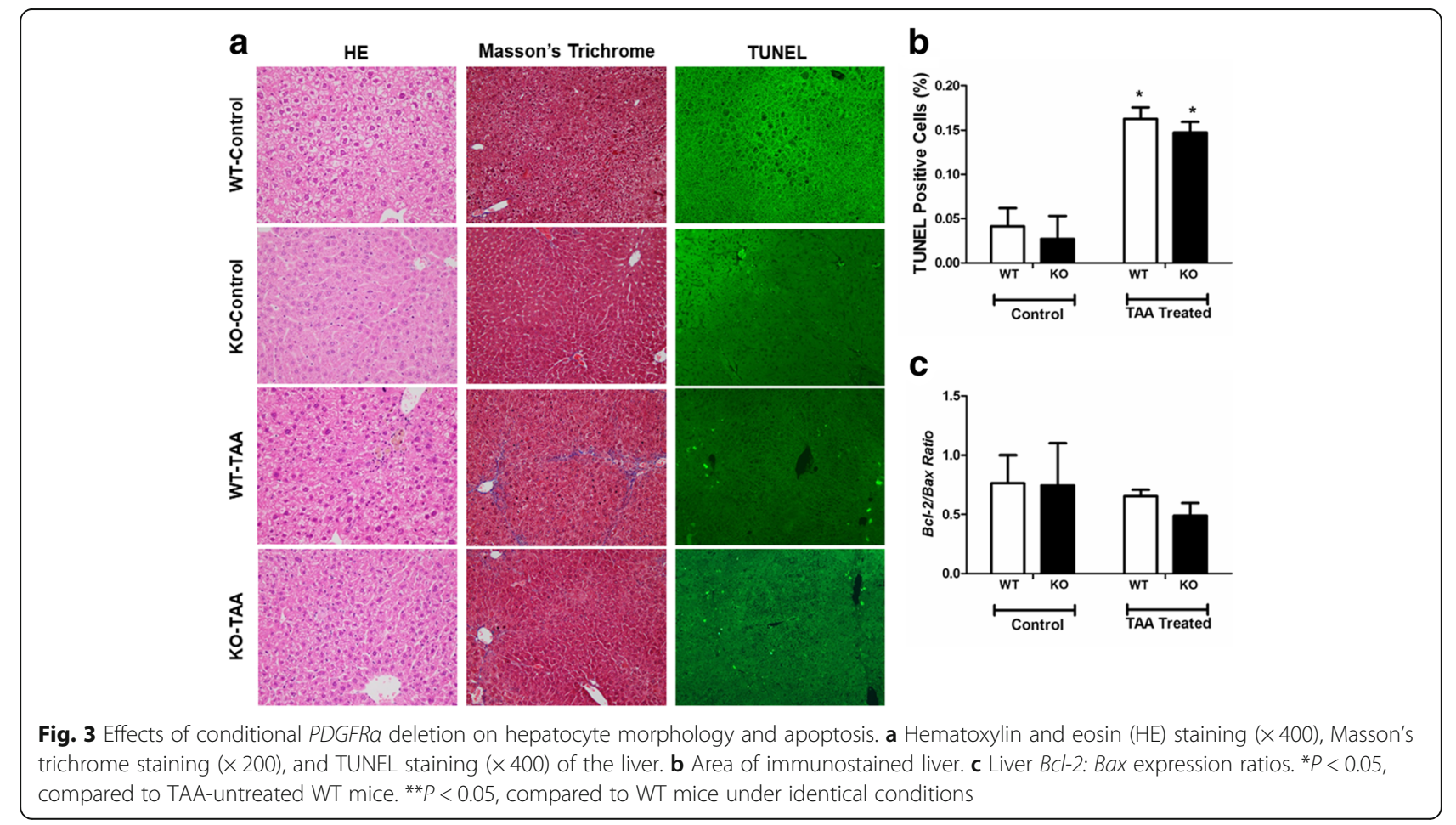


a
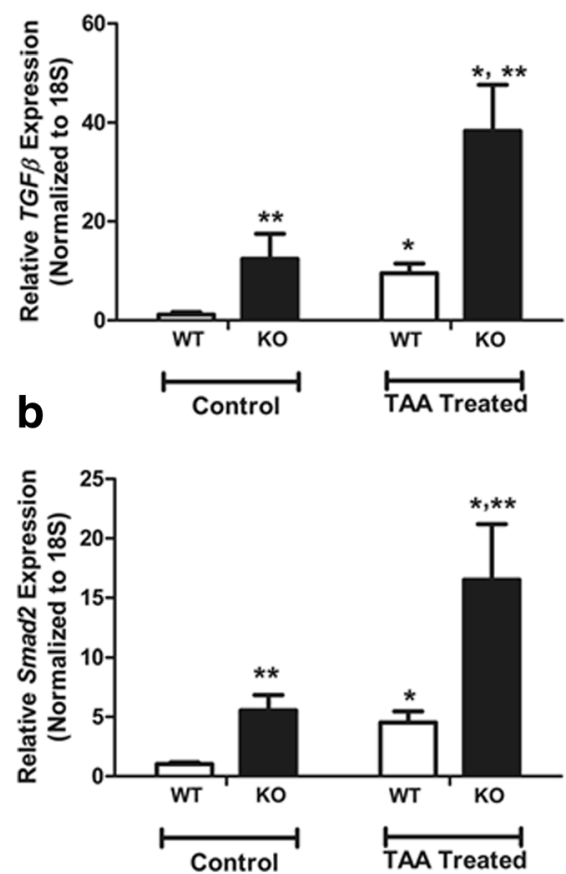

C

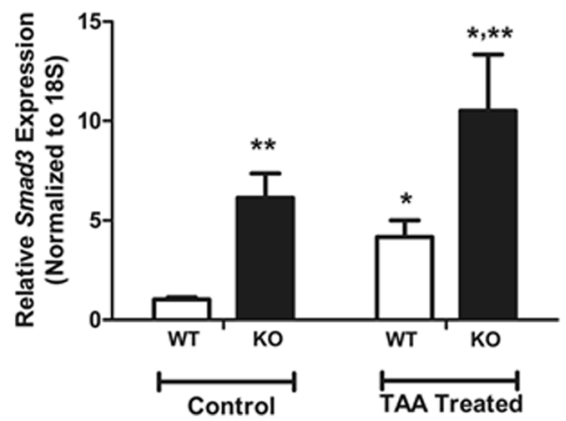

d
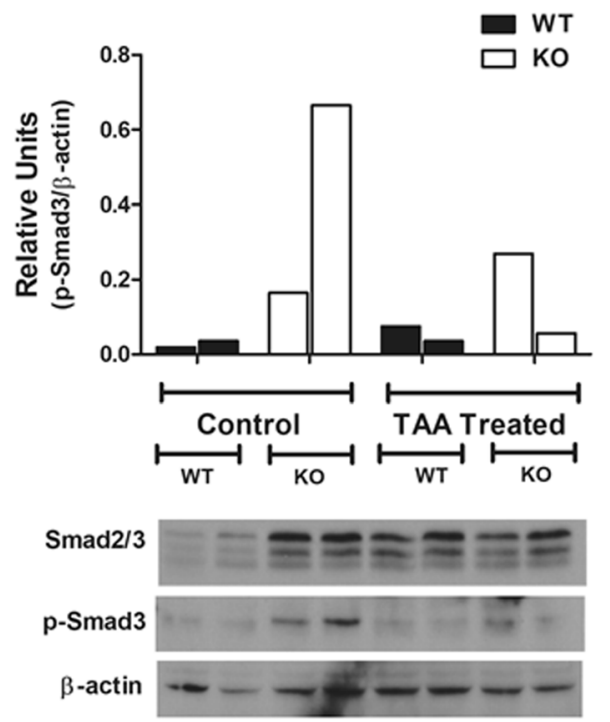

e

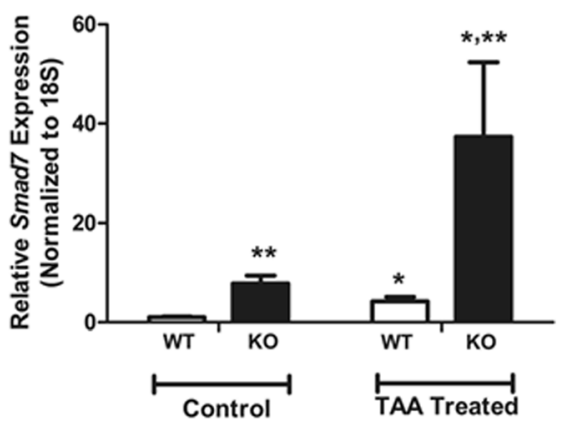

Fig. 4 Effect of hepatocyte PDGFRa deletion on TGF- $\beta$ signaling in TAA-treated mouse liver. Expression of (a) TGF $\beta$ (b), Smad2 (c), and Smad3. d Western blotting analyses of Smads; densitometry values normalized to that of $\beta$-actin are shown. e Expression of inhibitory $S m a d 7 .{ }^{*} P<0.05$, compared to TAA-untreated WT mice. ${ }^{* *} P<0.05$, compared to WT mice under identical conditions

TGF $\beta$ signaling in LX2 cells co-cultured with PDGFRasilenced Hep3B cells

LX2 cells cultured with PDGFR $\alpha$-silenced Hep3B cells exhibited attenuated expression of TGF $\beta$, and Smad2/3 (Fig. 6e). However, expression of $S m a d 7$ was not downregulated by co-culture with PDGFR $\alpha$-silenced Hep3B cells (Fig. 6e).

\section{PDGF ligands in PDGFRa-silenced Hep3B cell co-culture medium}

The levels of PDGF-BB and PDGF-CC were significantly reduced in the co-culture medium of LX2 cells with PDGFR $\alpha$-silenced Hep3B cells, and the reduction in the PDGF-BB level was of greater magnitude than that in the PDGF-CC level (Fig. 7).
PDGFRa expression in human liver with hepatitis without advanced fibrosis

Expression of PDGFR $\alpha$ was examined in normal human liver and human liver with hepatitis but not advanced fibrosis. Immunohistochemistry showed expression of PDGFR $\alpha$ at the hepatocyte membrane and in nonparenchymal cells in the liver with hepatitis, whereas the normal liver exhibited no PDGFR $\alpha$ staining (Fig. 8a).

Whole-cell lysates from livers with pathologically proven hepatitis but not advanced fibrosis $(n=7)$ were examined for $\operatorname{col} 1 \alpha(I)$ and PDGFR $\alpha$ expression and compared to that in normal liver tissue $(n=7)$. Livers with hepatitis but not significant liver fibrosis showed elevated PDGFR $\alpha$ expression compared to the normal livers, but $\operatorname{col} 1 \alpha(I)$ expression was not significantly different (Fig. 8b). 


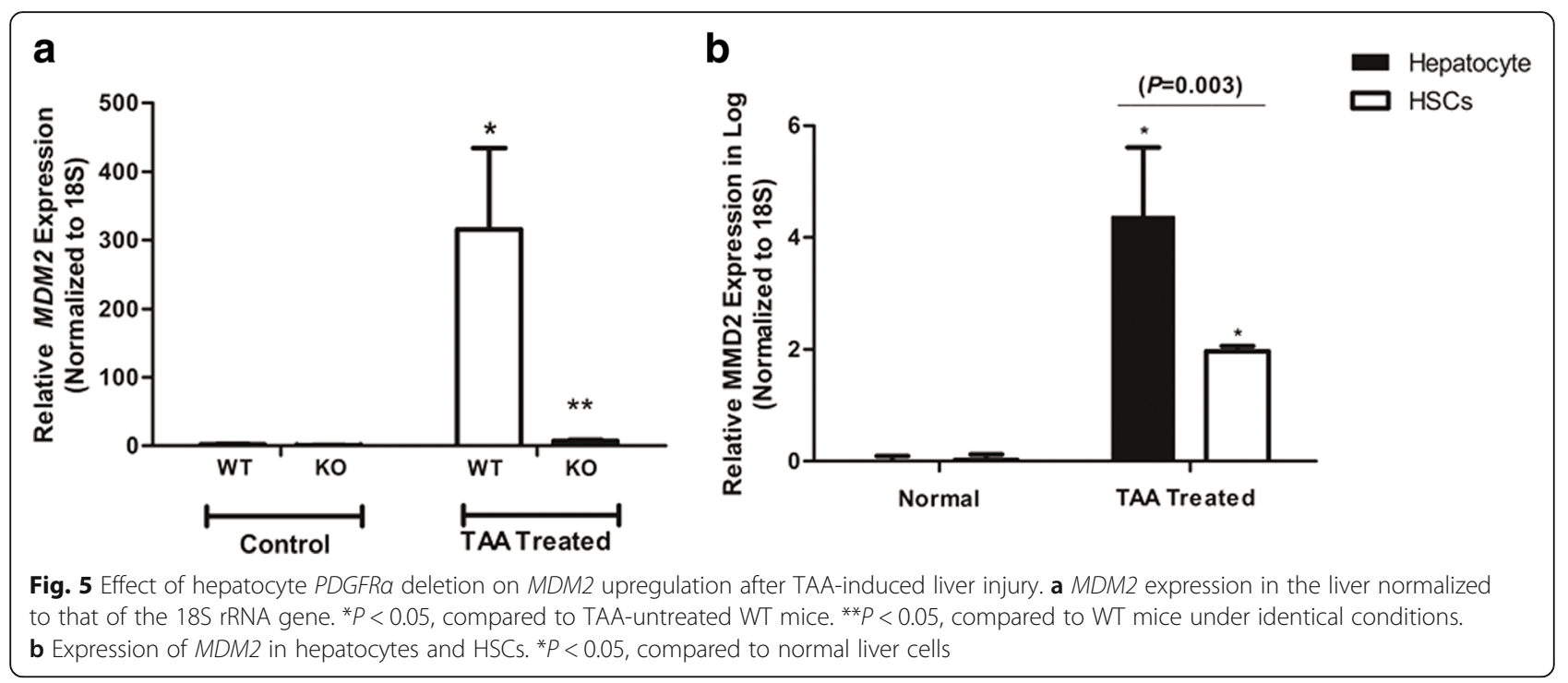

\section{Discussion}

PDGFR $\alpha$ expression is markedly elevated in chronic liver injury and enhanced PDGFR $\alpha$ activation contributes to liver fibrosis [19, 34]. The proliferation of PDGFR $\alpha$-positive HSCs during liver fibrosis can be suppressed by blocking of PDGFR $\alpha$ in HSCs [24]. While only PDGFR $\alpha$ in HSCs has been thought to play a role in liver fibrosis, we report that PDGFR $\alpha$ is upregulated in injured hepatocytes, which contributes to HSC proliferation, resulting in liver fibrosis. Deletion of PDGFR $\alpha$ in hepatocytes significantly attenuated TAA-induced liver fibrosis. PDGFR $\alpha$ in hepatocytes plays an important role in liver fibrosis by inducing HSC activation and proliferation; in vitro, HSCs co-cultured with PDGFR $\alpha$-deleted hepatocytes exhibited attenuated activation and decreased collagen production.

Normal adult hepatocytes express a low level of $P D G F R \alpha$, the role of which in hepatocytes in liver fibrosis was unclear. However, PDGFR $\alpha$ is reportedly expressed in cancerous hepatocytes, and facilitates the proliferation and migration of HCC cells, which are related to invasion

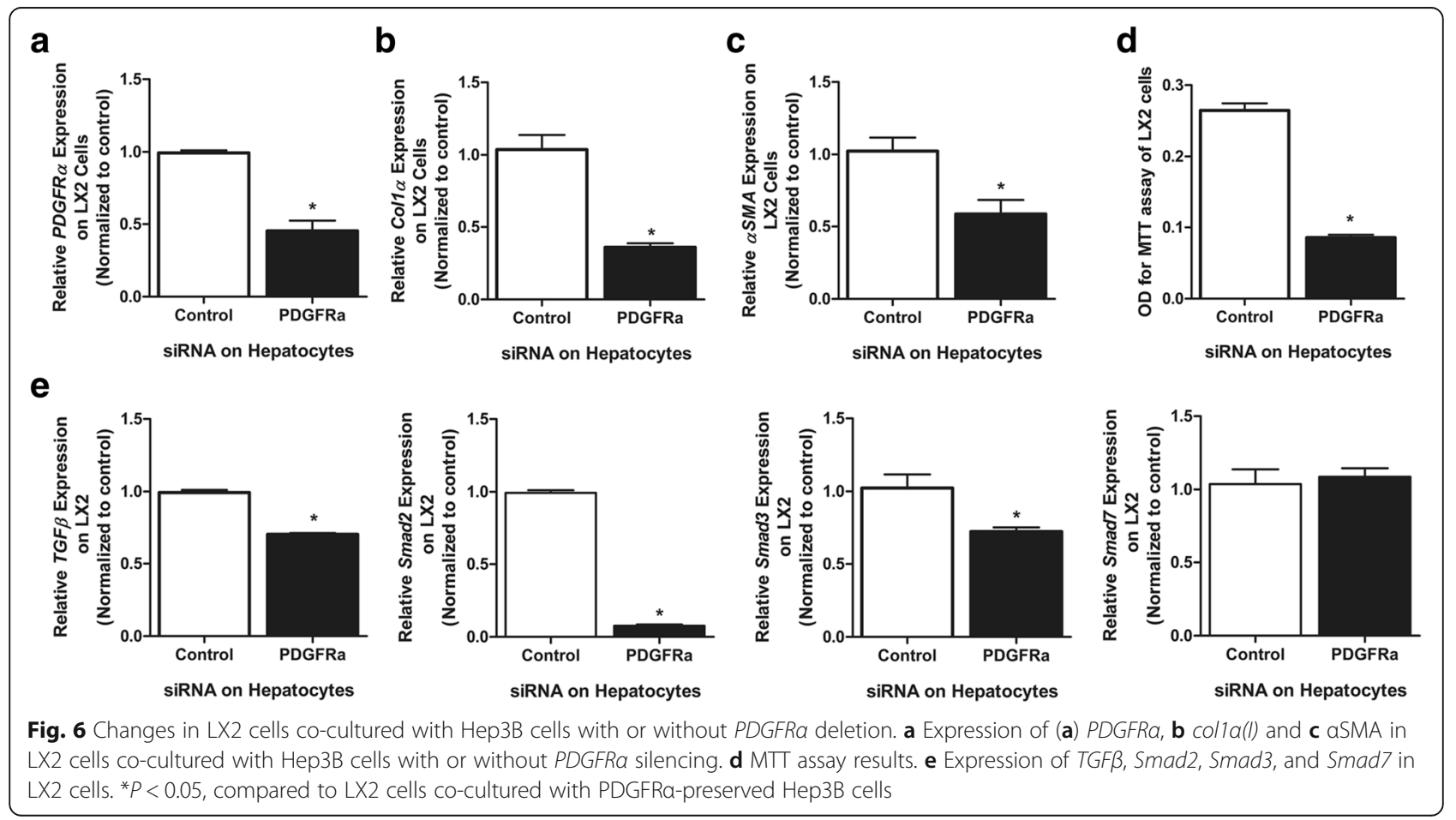



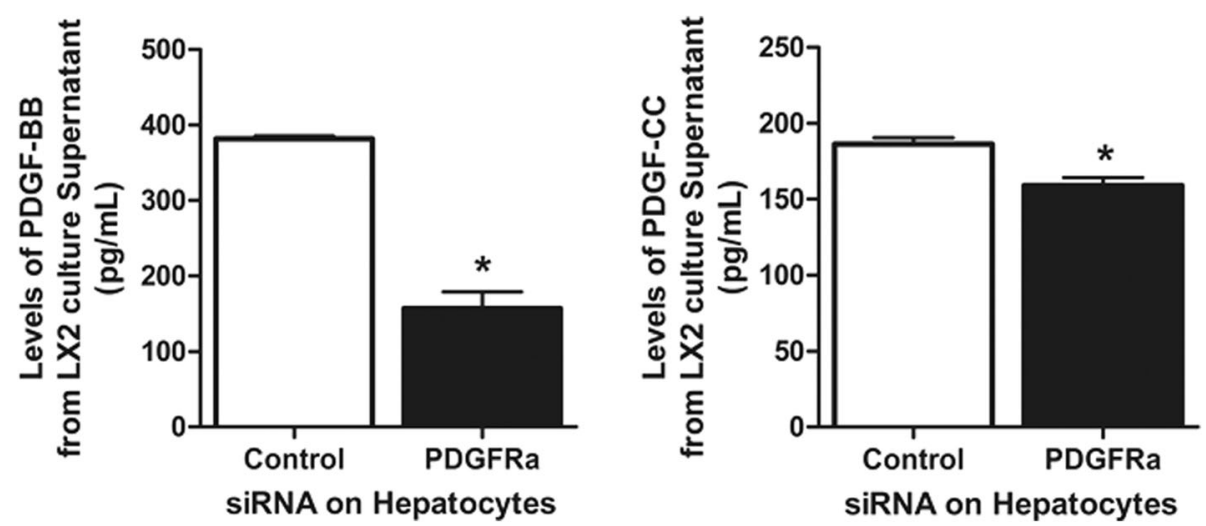

Fig. 7 Levels of PDGF-BB and -CC in Hep3B and LX2 co-culture medium. ${ }^{*} P<0.05$, compared to LX2 cells co-cultured with PDGFRa-preserved Hep3B cells

and metastasis [44, 45]. Hepatocytes expressing dominantnegative PDGFR $\alpha$ revealed decreased TGF- $\beta$-induced migration and tumor formation [44]. Therefore, our data suggest that PDGFR $\alpha$ in injured hepatocytes also contributes to liver fibrosis, the most important risk factor for HCC.

siRNA KO of PDGFR in hepatocytes resulted in decreased PDGF-BB and PDGF-CC levels in co-culture medium compared to co-culture of non-KO hepatocytes. Because activated HSCs are possible sources of PDGF ligands [46], and HSC activation was attenuated by PDGFR $\alpha$ deletion in hepatocytes, the reduced PDGF-BB and PDGF-CC levels were likely due to decreased production by HSCs. In addition, the decrement in the PDGF-BB level was of greater magnitude than that in the PDGF-CC level. PDGF-BB is reportedly the most potent mitogen for HSC activation, and some animal models of fibrosis, such as the bile-duct ligation model, show upregulation of PDGF-BB and PDGF-CC, but the latter to a lesser extent [47]. The mechanism by which PDGFRs on hepatocytes are activated is unclear. Increased ERK activation was detected in the livers of TAA-treated WT and KO mice, suggesting that PDGF ligands mediate PDGFR activation upon liver insult; the PDGFR $\alpha \mathrm{KO}$ mice exhibited attenuated ERK activation. However, upregulation of $M D M 2$, which is activated at the time of PDGF-independent PDGFR activation, was almost completely blocked by PDGFR $\alpha$ deletion in hepatocytes when WT mice liver showed upregulation of $M D M 2$ after TAA treatment.

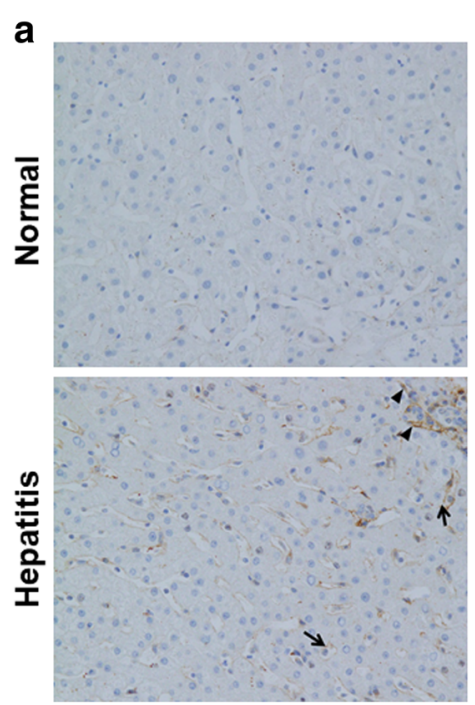

b

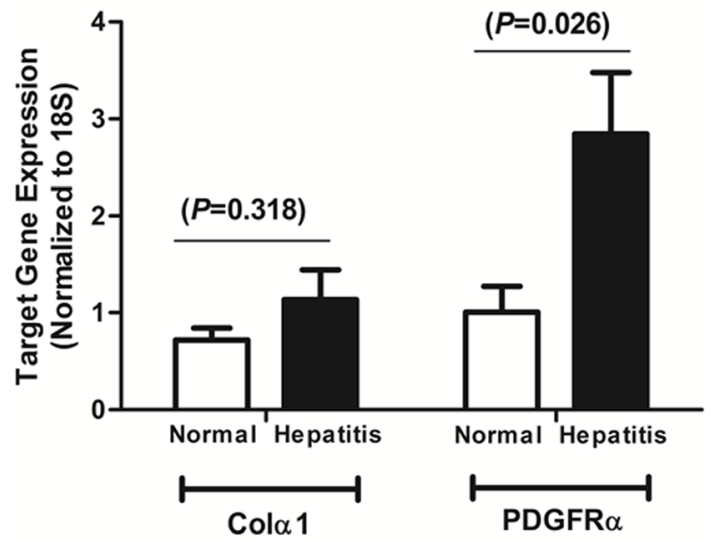

Fig. 8 Assessment of PDGFRa expression in the human liver with hepatitis without liver fibrosis. a Immunohistochemistry of liver specimens with hepatitis showed the presence of PDGFRa at the hepatocyte membrane (arrows) and in nonparenchymal cells (arrowheads), whereas normal-liver specimens showed no PDGFRa staining. Original magnification, $\times 400$. $\mathbf{b}$ Whole-cell lysates from pathologically proven hepatitis tissue without significant fibrosis $(n=7)$ were examined for colla(l) and PDGFRa mRNA levels compared to those in normal liver tissue $(n=7)$ 
Although both hepatocytes and HSCs from TAA-treated livers had elevated $M D M 2$ expression, the upregulation in hepatocytes was about one thousand-fold higher than that in HSCs. Therefore, PDGFR $\alpha$ in hepatocytes might be predominantly activated by non-PDGF mediated mechanisms; this warrants further research.

Elevated levels of TGF $\beta$ are seen in liver fibrosis and TGF $\beta$ overexpression results in liver fibrosis [48, 49]. TGF $\beta$ binds to cell surface receptors to initiate intracellular signal transduction pathways, including activation of Smad proteins [50-52]. Smad2 and Smad3 form complexes with Smad4 to activate gene expression. Smad7, an inhibitory Smad, disrupts receptor activation of Smad2/3, which inhibits TGF $\beta$ signaling in a negative feedback loop [53-55]. Activation of PDGFR $\alpha$ ligands by excess PDGF-C upregulates TGF $\beta / \mathrm{Smad} 3$ signaling to facilitate liver fibrosis and blocking of Smad3 signaling attenuates PDGF-C-induced liver fibrosis [38]. Deletion of PDGFR $\alpha$ in hepatocytes results in upregulation of $T G F \beta$ and $S m a d 2 / 3$ expression in the whole liver. However, this increase in $T G F \beta$ and $S m a d 2 / 3$ expression is offset by overexpression of $\operatorname{Smad7}$, resulting in attenuation of TAA-induced liver fibrosis. The cellular source of elevated TGF $\beta$, Smad2/3, and Smad7 in the liver after hepatocyte $P D G F R \alpha$ deletion is unclear. However, co-culture of PDGFR $\alpha$-deleted hepatocytes and normal HSCs resulted in significant upregulation of TGF $\beta$ and Smad2/3 expression in HSCs and maintenance of Smad7 expression.

Particles of injured hepatocytes, as apoptotic bodies, promote secretion of proinflammatory and fibrogenic cytokines from neighboring inflammatory cells such as macrophages [56]. In addition, damage-associated molecular patterns (DAMPs) have been suggested to be released from injured hepatocytes; this may induce liver fibrosis [57]. It can be speculated that PDGFR $\alpha$ in injured hepatocytes induces liver fibrosis by promoting the release of DAMPs and activating HSCs. Although PDGR $\alpha$-deleted HCC cells exhibit enhanced apoptosis [43], this was not so for non-cancerous hepatocytes in this study.

\section{Conclusions}

Our findings suggest that although PDGFR expression in normal hepatocytes is negligible, it is upregulated by liver insult and plays a vital role in liver fibrosis by facilitating the activation and proliferation of HSCs.

\footnotetext{
Abbreviations

Alb: Albumin; Col1a(l): Collagen 1a(l); DAMP: Damage-associated molecular pattern; ECM: Extracellular matrix; HCC: Hepatocellular carcinoma; HSC: Hepatic stellate cell; KO: Knockout; MDM2: Mouse double minute 2; MTT: Methyl thiazolyl tetrazolium; PDGF: Platelet-derived growth factor; PDGFR: Platelet-derived growth factor receptor; TAA: Thioacetamide; Tg: Transgenic; TGFß: Transforming growth factor beta; TUNEL: Terminal dUTP nick end-labeling; WT: Wild-type; aSMA: Alpha-smooth muscle actin
}

Acknowledgments

Not applicable.

\section{Funding}

This research was supported by the Basic Science Research Program through the National Research Foundation of Korea (NRF) funded by the Ministry of Science, ICT \& Future Planning (NRF-2016R1A2B4015192), and the NextGeneration 21 Program (PJ01323001).

\section{Availability of data and materials}

All data generated or analyzed during this study are included in this article.

\section{Authors' contributions}

$J I L$ conceived and designed experiments. BJL, WKL, HWL, and KSL contributed to experimental planning and performed the experiments. HYC performed the experiments. HWL, JKK, and HYC analyzed the data. BJL and WKL drafted the manuscript. All authors read and approved the final manuscript.

\section{Ethics approval and consent to participate}

All experiments involving mice were approved by the Institutional Animal Use and Care Committee (IACUC) at Gangnam Severance Hospital, Yonsei University College of Medicine (permit number 0031) in accordance with the recommendations and restrictions of the IACUC, which follows the National Institutes of Health guidelines. Use of human specimens was approved by the Institutional Review Board of Gangnam Severance Hospital, Yonsei University College of Medicine, Seoul, Republic of Korea (3-2015-0045). The liver specimens were archived at the Tissue Bank of Yonsei University College of Medicine, Gangnam Severance Hospital after acquiring patient consent.

\section{Consent for publication}

This work does not contain individual patient's personal details or information.

\section{Competing interests}

The authors declare that they have no competing interests.

\section{Publisher's Note}

Springer Nature remains neutral with regard to jurisdictional claims in published maps and institutional affiliations.

\section{Author details}

${ }^{1}$ Department of Pathology, Gangnam Severance Hospital, Yonsei University College of Medicine, Seoul, Republic of Korea. '2Laboratory of Developmental Genetics, Department of Biomedical Sciences, Inha University College of Medicine, Incheon, Republic of Korea. ${ }^{3}$ Department of Internal Medicine, Gangnam Severance Hospital, Yonsei University College of Medicine, Seoul, Republic of Korea. ${ }^{4}$ Medical Research Center, Gangnam Severance Hospital, Seoul, South Korea.

Received: 3 August 2018 Accepted: 21 November 2018

Published online: 03 December 2018

References

1. Friedman SL. Mechanisms of hepatic fibrogenesis. Gastroenterology. 2008; 134:1655-69.

2. Kisseleva T, Brenner DA. Role of hepatic stellate cells in fibrogenesis and the reversal of fibrosis. J Gastroenterol Hepatol. 2007;22(Suppl 1):S73-8.

3. Pellicoro A, Ramachandran P, Iredale JP. Reversibility of liver fibrosis. Fibrogenesis Tissue Repair. 2012;5(Suppl 1):S26.

4. Wells RG. Cellular sources of extracellular matrix in hepatic fibrosis. Clin Liver Dis. 2008;12:759-68 viii.

5. Mederacke I. Liver fibrosis: mouse models and relevance in human liver diseases. Z Gastroenterol. 2013;51:55-62.

6. Lim YS, Kim WR. The global impact of hepatic fibrosis and end-stage liver disease. Clin Liver Dis. 2008;12:733-46 vii.

7. Pinzani M, Vizzutti F. Fibrosis and cirrhosis reversibility: clinical features and implications. Clin Liver Dis. 2008;12:901-13 x.

8. Severi T, van Malenstein H, Verslype C, van Pelt JF. Tumor initiation and progression in hepatocellular carcinoma: risk factors, classification, and therapeutic targets. Acta Pharmacol Sin. 2010;31:1409-20.

9. Bataller R, Brenner DA. Liver fibrosis. J Clin Invest. 2005;115:209-18. 
10. Hernandez-Gea V, Friedman SL. Pathogenesis of liver fibrosis. Annu Rev Pathol. 2011;6:425-56.

11. Oseini AM, Roberts LR. PDGFRalpha: a new therapeutic target in the treatment of hepatocellular carcinoma? Expert Opin Ther Targets. 2009;13: 443-54.

12. Heldin $\mathrm{CH}$. Targeting the PDGF signaling pathway in tumor treatment. Cell Commun Signal. 2013;11:97.

13. Zymek P, Bujak M, Chatila K, Cieslak A, Thakker G, Entman ML, Frangogiannis NG. The role of platelet-derived growth factor signaling in healing myocardial infarcts. J Am Coll Cardiol. 2006;48:2315-23.

14. Abdollahi A, Li M, Ping G, Plathow C, Domhan S, Kiessling F, Lee LB, McMahon G, Grone HJ, Lipson KE, et al. Inhibition of platelet-derived growth factor signaling attenuates pulmonary fibrosis. J Exp Med. 2005;201: 925-35.

15. Chen YT, Chang FC, Wu CF, Chou YH, Hsu HL, Chiang WC, Shen J, Chen YM, Wu KD, Tsai TJ, et al. Platelet-derived growth factor receptor signaling activates pericyte-myofibroblast transition in obstructive and post-ischemic kidney fibrosis. Kidney Int. 2011;80:1170-81.

16. Kocabayoglu P, Lade A, Lee YA, Dragomir AC, Sun X, Fiel MI, Thung S, Aloman C, Soriano P, Hoshida Y, et al. Beta-PDGF receptor expressed by hepatic stellate cells regulates fibrosis in murine liver injury, but not carcinogenesis. J Hepatol. 2015;63:141-7.

17. Breitkopf K, Roeyen C, Sawitza I, Wickert L, Floege J, Gressner AM. Expression patterns of PDGF-A, - B, -C and -D and the PDGF-receptors alpha and beta in activated rat hepatic stellate cells (HSC). Cytokine. 2005;31:349-57.

18. Iwayama T, Olson LE. Involvement of PDGF in fibrosis and scleroderma: recent insights from animal models and potential therapeutic opportunities. Curr Rheumatol Rep. 2013;15:304.

19. Hayes BJ, Riehle KJ, Shimizu-Albergine M, Bauer RL, Hudkins KL, Johansson F, Yeh MM, Mahoney WM Jr, Yeung RS, Campbell JS. Activation of plateletderived growth factor receptor alpha contributes to liver fibrosis. PLoS One. 2014;9:e92925.

20. Pinzani M. Platelet-derived growth factor receptor expression in hepatic stellate cells: how too much of a good thing can be bad. Hepatology. 1995; 22:997-9.

21. Wong L, Yamasaki G, Johnson RJ, Friedman SL. Induction of beta-plateletderived growth factor receptor in rat hepatic lipocytes during cellular activation in vivo and in culture. J Clin Invest. 1994:94:1563-9.

22. Thieringer F, Maass T, Czochra P, Klopcic B, Conrad I, Friebe D, Schirmacher P, Lohse AW, Blessing M, Galle PR, et al. Spontaneous hepatic fibrosis in transgenic mice overexpressing PDGF-A. Gene. 2008;423:23-8.

23. Czochra P, Klopcic B, Meyer E, Herkel J, Garcia-Lazaro JF, Thieringer F, Schirmacher P, Biesterfeld S, Galle PR, Lohse AW, et al. Liver fibrosis induced by hepatic overexpression of PDGF-B in transgenic mice. J Hepatol. 2006;45: 419-28.

24. Kikuchi A, Pradhan-Sundd T, Singh S, Nagarajan S, Loizos N, Monga S. Platelet-derived growth factor receptor alpha contributes to human hepatic stellate cell proliferation and migration. Am J Pathol. 2017;187:2273-87.

25. Campbell JS, Hughes SD, Gilbertson DG, Palmer TE, Holdren MS, Haran AC, Odell MM, Bauer RL, Ren HP, Haugen HS, et al. Platelet-derived growth factor $C$ induces liver fibrosis, steatosis, and hepatocellular carcinoma. Proc Natl Acad Sci U S A. 2005;102:3389-94.

26. Campbell JS, Johnson MM, Bauer RL, Hudkins KL, Gilbertson DG, Riehle KJ Yeh MM, Alpers CE, Fausto N. Targeting stromal cells for the treatment of platelet-derived growth factor C-induced hepatocellular carcinogenesis. Differentiation. 2007;75:843-52.

27. Awuah PK, Nejak-Bowen KN, Monga SPS. Role and regulation of PDGFR alpha signaling in liver development and regeneration. Am J Pathol. 2013; 182:1648-58.

28. Yu JH, Kim JM, Kim JK, Choi SJ, Lee KS, Lee JW, Chang HY, Lee Jl. Plateletderived growth factor receptor alpha in hepatocellular carcinoma is a prognostic marker independent of underlying liver cirrhosis. Oncotarget. 2017:8:39534-46.

29. Seglen PO. Preparation of isolated rat liver cells. Methods Cell Biol. 1976;13: 29-83.

30. O'Brien MJ, Keating NM, Elderiny S, Cerda S, Keaveny AP, Afdhal NH, Nunes DP. An assessment of digital image analysis to measure fibrosis in liver biopsy specimens of patients with chronic hepatitis C. Am J Clin Pathol. 2000;114:712-8

31. Friedman SL, Rockey DC, McGuire RF, Maher JJ, Boyles JK, Yamasaki G. Isolated hepatic lipocytes and Kupffer cells from normal human liver: morphological and functional characteristics in primary culture. Hepatology. 1992;15:234-43.

32. Hiron M, Daveau M, Arnaud P, Bauer J, Lebreton JP. The human hepatoma Hep3B cell line as an experimental model in the study of the long-term regulation of acute-phase proteins by cytokines. Biochem J. 1992;287(Pt 1): 255-9.

33. Livak KJ, Schmittgen TD. Analysis of relative gene expression data using real-time quantitative PCR and the 2(T)(-Delta Delta C) method. Methods. 2001;25:402-8.

34. Borkham-Kamphorst E, Kovalenko E, van Roeyen CR, Gassler N, Bomble M, Ostendorf T, Floege J, Gressner AM, Weiskirchen R. Platelet-derived growth factor isoform expression in carbon tetrachloride-induced chronic liver injury. Lab Investig. 2008;88:1090-100.

35. Kazlauskas A. PDGFs and their receptors. Gene. 2017;614:1-7.

36. Ikeguchi M, Hirooka Y, Kaibara N. Quantitative analysis of apoptosis-related gene expression in hepatocellular carcinoma. Cancer. 2002;95:1938-45.

37. Oltvai ZN, Milliman CL, Korsmeyer SJ. BCl-2 heterodimerizes in vivo with a conserved homolog, Bax, that accelerates programmed cell death. Cell. 1993;74:609-19.

38. Lee Jl, Wright JH, Johnson MM, Bauer RL, Sorg K, Yuen S, Hayes BJ, Nguyen $\mathrm{L}$, Riehle KJ, Campbell JS. Role of Smad3 in platelet-derived growth factor-Cinduced liver fibrosis. Am J Physiol Cell Physiol. 2016;310:C436-45.

39. Martin K, Trouche D, Hagemeier C, Sorensen TS, La Thangue NB, Kouzarides T. Stimulation of E2F1/DP1 transcriptional activity by MDM2 oncoprotein. Nature. 1995;375:691-4.

40. Momand J, Zambetti GP. Mdm-2: "big brother" of p53. J Cell Biochem. 1997. 64:343-52.

41. Lei H, Velez G, Kazlauskas A. Pathological signaling via platelet-derived growth factor receptor \{alpha\} involves chronic activation of Akt and suppression of p53. Mol Cell Biol. 2011;31:1788-99.

42. Pennock S, Haddock LJ, Eliott D, Mukai S, Kazlauskas A. Is neutralizing vitreal growth factors a viable strategy to prevent proliferative vitreoretinopathy? Prog Retin Eye Res. 2014;40:16-34.

43. Stock P, Monga D, Tan X, Micsenyi A, Loizos N, Monga SP. Platelet-derived growth factor receptor-alpha: a novel therapeutic target in human hepatocellular cancer. Mol Cancer Ther. 2007;6:1932-41.

44. Gotzmann J, Fischer AN, Zojer M, Mikula M, Proell V, Huber H, Jechlinger M, Waerner T, Weith A, Beug H, et al. A crucial function of PDGF in TGF-betamediated cancer progression of hepatocytes. Oncogene. 2006;25:3170-85.

45. Fischer AN, Fuchs E, Mikula M, Huber H, Beug H, Mikulits W. PDGF essentially links TGF-beta signaling to nuclear beta-catenin accumulation in hepatocellular carcinoma progression. Oncogene. 2007;26:3395-405.

46. Greuter T, Shah VH. Hepatic sinusoids in liver injury, inflammation, and fibrosis: new pathophysiological insights. J Gastroenterol. 2016;51:511-9.

47. Borkham-Kamphorst E, Weiskirchen R. The PDGF system and its antagonists in liver fibrosis. Cytokine Growth Factor Rev. 2016;28:53-61.

48. Sanderson N, Factor V, Nagy P, Kopp J, Kondaiah P, Wakefield L, Roberts AB, Sporn MB, Thorgeirsson SS. Hepatic expression of mature transforming growth factor beta 1 in transgenic mice results in multiple tissue lesions. Proc Natl Acad Sci U S A. 1995:92:2572-6.

49. Kanzler S, Lohse AW, Keil A, Henninger J, Dienes HP, Schirmacher P, RoseJohn S, zum Buschenfelde KH, Blessing M. TGF-beta1 in liver fibrosis: an inducible transgenic mouse model to study liver fibrogenesis. Am J Phys. 1999;276:G1059-68.

50. Dooley S, ten Dijke P. TGF-beta in progression of liver disease. Cell Tissue Res. 2012;347:245-56.

51. Inagaki $Y$, Okazaki I. Emerging insights into transforming growth factor beta Smad signal in hepatic fibrogenesis. Gut. 2007;56:284-92

52. Wells RG. Fibrogenesis. V. TGF-beta signaling pathways. Am J Physiol Gastrointest Liver Physiol. 2000;279:G845-50.

53. Lonn P, Moren A, Raja E, Dahl M, Moustakas A. Regulating the stability of TGFbeta receptors and Smads. Cell Res. 2009;19:21-35.

54. Moustakas A, Heldin $\mathrm{CH}$. The regulation of TGFbeta signal transduction. Development. 2009;136:3699-714.

55. Moustakas A, Souchelnytskyi S, Heldin CH. Smad regulation in TGF-beta signal transduction. J Cell Sci. 2001;114:4359-69.

56. Seki E, Schwabe RF. Hepatic inflammation and fibrosis: functional links and key pathways. Hepatology. 2015;61:1066-79.

57. Luedde T, Kaplowitz N, Schwabe RF. Cell death and cell death responses in liver disease: mechanisms and clinical relevance. Gastroenterology. 2014;147: 765-83 e764. 